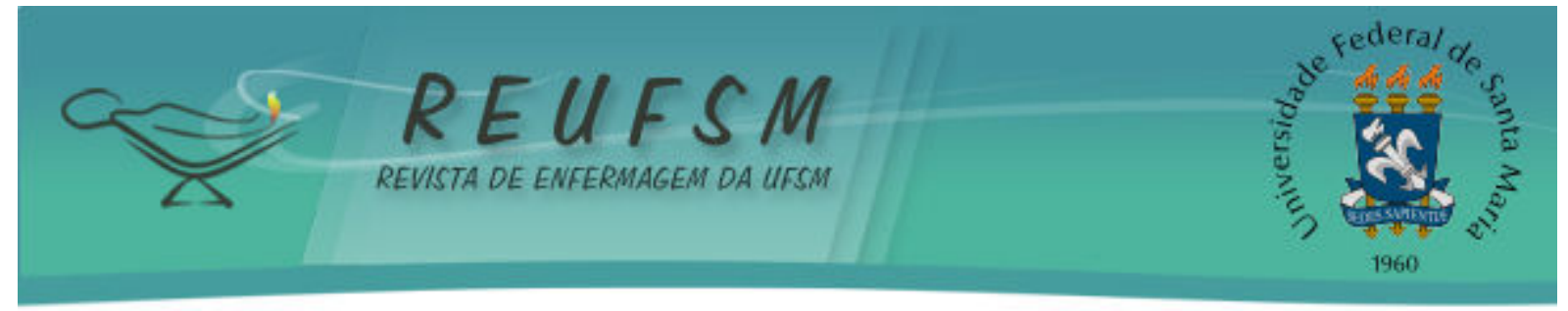

ARTIGO ORIGINAL

\title{
A ESCOLHA PELO ATENDIMENTO EM CASA DE PARTO E AVALIAÇÃO DO CUIDADO PRÉ- NATAL
}

\section{THE CHOICE FOR ATTENDANCE IN THE BIRTH CENTER AND EVALUATION OF PRENATAL CARE}

\section{LA OPCIÓN DEL ATENDIMIENTO EN CASA DE PARTO Y EVALUACIÓN DEL CUIDADO PRENATAL}

Doi: $10.5902 / 2179769213268$

Adriana Lenho de Figueiredo Pereira ${ }^{1}$ Marina Caldas Nicácio

RESUMO: Objetivos: a presente pesquisa objetivou identificar os motivos da escolha pelo atendimento na Casa de Parto e avaliar a satisfação das gestantes com o cuidado prénatal. Método: estudo descritivo de abordagem quantitativa e baseado na técnica da pesquisa de opinião. Cinquenta e uma mulheres responderam questionário estruturado a partir das cinco respostas da Escala de Likert. Os dados receberam tratamento estatístico descritivo e foi calculado o ranking médio. Resultados: as mulheres optaram pela Casa de Parto devido ao ambiente acolhedor, incentivo ao parto normal e garantia da presença do acompanhante e da internação no momento do parto. Elas têm alto nível de satisfação com o cuidado pré-natal, com ranking médio acima de quatro em quase todas as variáveis investigadas. Conclusão: a atenção e a cordialidade dos profissionais, a privacidade e o conforto do ambiente de cuidado promoveram a satisfação com o cuidado pré-natal em Casa de Parto.

Descritores: Cuidado pré-natal; Qualidade da assistência à saúde; Enfermagem obstétrica; Centros independentes de assistência à gravidez e ao parto.

ABSTRACT: Objectives: the purpose of the current investigation was to identify the reasons of the choice for attendance in free-standing Birthing Center and evaluate the satisfaction of pregnant women with antenatal care. Method: quantitative descriptive study, based on the survey research. Fifty-one women answered the structured questionnaire that used the five-point Likert-type scale. The data analysis utilized descriptive statistics and the mean ranking was calculated. Results: the women have chosen the Birthing Center assistance due to its welcoming atmosphere, the encouragement to normal birth and the guarantee of the presence of support person and admission for the moment of delivery. They have a high level of satisfaction with antenatal care, with mean ranking above four in almost all investigated variables. Conclusion: the cordiality and professional attention, privacy and comfort of the care environment promoted the satisfaction with prenatal care in Birthing Center.

Descriptors: Prenatal care; Quality of health care; Obstetric nursing; Birthing centers.

RESUMEN: Objetivos: la presiente investigación objetivó identificar los motivos de la opción por el atendimiento en Casa de Parto y evaluar la satisfacción de las embarazadas con el cuidado prenatal. Método: estudio descriptivo, cuantitativo, basado en estudios de opinión. Cincuenta y una mujeres respondieron cuestionario estructurado a partir de las

\footnotetext{
${ }^{1}$ Enfermeira obstétrica, Doutora em Enfermagem, Docente do Departamento de Enfermagem Materno-Infantil, Faculdade de Enfermagem, Universidade do Estado do Rio de Janeiro/UERJ. Rio de Janeiro (RJ), Brasil. E-mail: adrianalenho.uerj@gmail.com

2 Acadêmica de Enfermagem. Bolsista de Iniciação Científica. Faculdade de Enfermagem, Universidade do Estado do Rio de Janeiro/UERJ. Rio de Janeiro (RJ), Brasil. E-mail: mari_kaldas@hotmail.com
} 


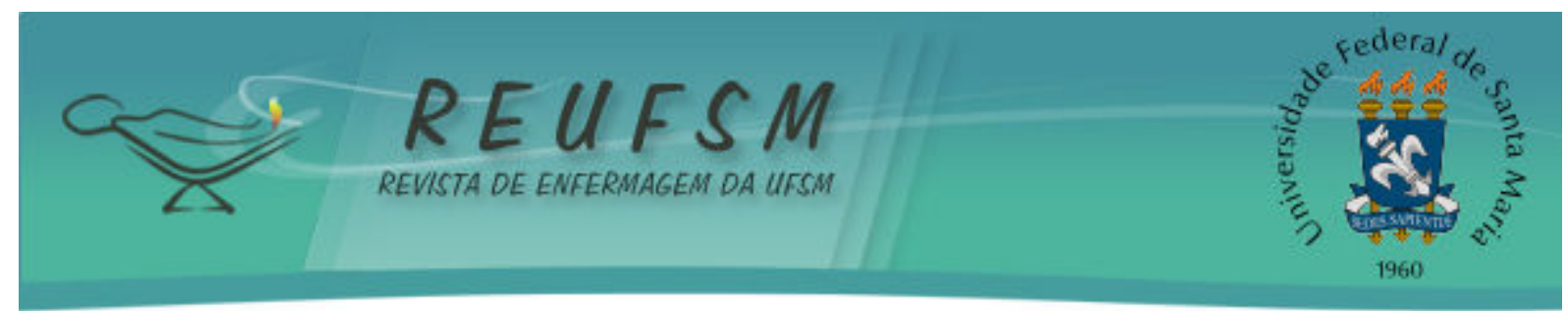

cinco respuestas de la Escala de Likert. Los datos fueron tratados por estadística descriptiva y se calculó el ranking medio. Resultados: las mujeres optaron por la Casa de Parto debido a su ambiente acogedor, promoción del parto normal, garantía de la presencia del acompañante y de la internación para el parto. Ellas tienen alto nivel de satisfacción con la atención prenatal, con ranking medio mayor que cuatro en casi todas las variables investigadas. Conclusión: la atención y cordialidad de los profesionales, privacidad y conforto del ambiente de cuidado promovieron la satisfacción con la atención prenatal en la Casa de Parto.

Descriptores: Atención prenatal; Calidad de la atención de salud; Enfermería obstétrica; Centros Independientes de Asistencia al Embarazo y al Parto.

\section{INTRODUÇÃO}

O modelo assistencial hegemônico nas maternidades brasileiras caracteriza-se pela medicalização, intervenção e impessoalidade na relação entre as mulheres grávidas e os profissionais de saúde. Estas características suscitaram questionamentos entre os atores integrantes do movimento da Reforma Sanitária, como os limites deste modelo para a garantia dos direitos de cidadania e a melhoria dos indicadores de saúde materna e neonatal, e, especialmente, do movimento organizado de mulheres, em torno de pautas dos direitos sexuais e reprodutivos, que intentavam estabelecer mudanças nas práticas assistenciais e nos serviços de saúde. ${ }^{1}$

Frente a esses questionamentos e às transformações nas políticas de saúde voltadas para a qualificação e humanização da assistência à gestação, parto e nascimento no país, em 1999, o Ministério da Saúde (MS) instituiu os Centros de Parto Normal (CPN) para mulheres cujas gestações são classificadas como de baixo risco obstétrico no Sistema Único de Saúde (SUS). Estes Centros podem estar situados no interior, próximos ou afastados da estrutura física do hospital. Há tendência de denominar como Casa de Parto os centros de parto que são fisicamente distanciados do hospital. ${ }^{1}$

A Secretaria Municipal de Saúde do Rio de Janeiro (SMS/RJ) criou um CPN isolado e comunitário na zona oeste da cidade em 2004, que foi denominado de Casa de Parto David Capistrano Filho, onde a assistência materna e neonatal é realizada por enfermeiras obstétricas. Esta unidade de saúde atende ao pré-natal, parto, pós-parto e as intercorrências obstétricas das gestantes cadastradas. Para que a gestante tenha seu parto atendido na Casa de Parto, é necessária a realização prévia do acompanhamento pré-natal na instituição. Cabe esclarecer, que pode haver referência de mulheres com até 34 semanas de gestação e que iniciaram o pré-natal na atenção básica da região adstrita. ${ }^{1}$

A resolução SMS/RJ n.1041/2004 estabelece que esse serviço de saúde é uma estratégia de ampliação, qualificação e humanização da assistência perinatal, que contribui na mudança do paradigma assistencial vigente, centrado no hospital, nos procedimentos de intervenção e no uso da tecnologia biomédica, em direção a um modelo de valorização dos aspectos sociais e emocionais do parto e nascimento. ${ }^{1}$

A valorização destes aspectos sociais e emocionais e as experiências internacionais exitosas nos países desenvolvidos motivaram a criação dos CPN no Brasil. Pesquisas internacionais identificaram que estas instituições promovem bons resultados perinatais e altos índices de satisfação materna. ${ }^{2-3}$

A satisfação dos usuários é um componente previsto no Programa Nacional de Avaliação de Serviços de Saúde (PNASS), que objetiva avaliar os serviços de saúde do SUS nas dimensões de estruturas, processos e resultados relacionados ao risco, acesso e satisfação dos cidadãos. ${ }^{4}$ 


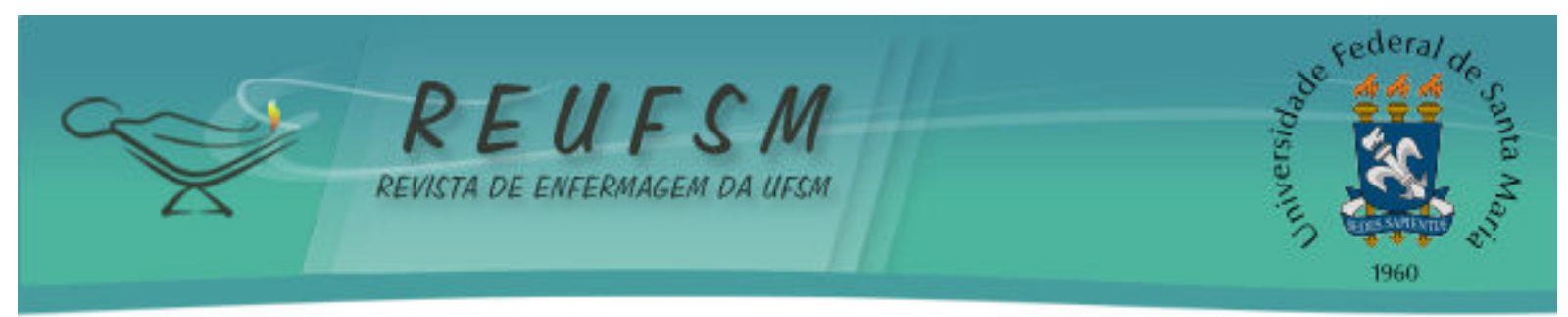

A avaliação é parte essencial para o planejamento e a gestão do sistema de saúde. A avaliação inclui a investigação de quatro componentes: 1) avaliação da estrutura, que se refere à adequação dos recursos físicos, humanos e organizacionais (comitês, protocolos assistenciais, etc.); 2) avaliação dos processos de trabalho nas áreas de gestão, serviços de apoio e serviços assistenciais, como a organização e documentação, protocolos, normas e rotinas; 3) avaliação dos resultados, que verifica o impacto da assistência prestada na situação de saúde, conhecimento e comportamento do paciente, que inclui os indicadores epidemiológicos; e, finalmente, 4) avaliação da satisfação dos pacientes em relação ao atendimento recebido e dos provedores destes serviços em relação aos seus ambientes de trabalho. ${ }^{4}$

No campo específico da atenção obstétrica, há o reconhecimento governamental de que os programas de saúde necessitam avançar na melhoria do acesso, na qualidade da assistência às gestantes no pré-natal, no parto e aos recém-nascidos, bem como nos indicadores de morbimortalidade materna e neonatal. ${ }^{5}$

A análise do cuidado às gestantes na atenção básica apontou para a persistência do modelo hegemônico, centrado nos procedimentos clínico-obstétricos e no modelo biomédico, e identificou a necessidade de avanços das práticas de promoção à saúde, de escuta ativa e de maior vínculo entre as gestantes e os profissionais. ${ }^{6}$

Em relação à consulta de enfermagem no pré-natal nesse nível de atenção, as mulheres avaliaram satisfatoriamente este atendimento e expressaram alto grau de satisfação em relação à assistência prestada. ${ }^{7}$ No entanto, outras pesquisas identificaram a necessidade de aprimoramentos em relação aos princípios da integralidade e da humanização da assistência, bem como nas atividades de educação em saúde sobre as mudanças fisiológicas da gravidez, trabalho de parto e parto, cuidados com a criança e amamentação. ${ }^{8 \cdot 10}$

Os CPN atendem à mulher somente no momento do parto. Na Casa de Parto, o acompanhamento pré-natal e a assistência ao parto estão integrados e estão sob responsabilidade técnica das enfermeiras obstétricas, constituindo-se um cenário assistencial singular e com cuidados contínuos à mulher no período gravídico-puerperal. Apenas um estudo tratou da avaliação da assistência pré-natal em Casa de Parto sob a perspectiva das mulheres. Neste estudo foi verificado que a assistência prestada nas consultas pré-natal foi bem avaliada pelas gestantes investigadas. ${ }^{11}$

Em consideração a essa singularidade organizacional e assistencial da Casa de Parto, estabeleceu-se os seguintes questionamentos: Por que as mulheres optaram pelo atendimento obstétrico na Casa de Parto? Qual o nível de satisfação destas mulheres com o cuidado pré-natal prestado? Para responder estas questões, foi elaborada a presente pesquisa que objetivou identificar os motivos da escolha pelo atendimento na Casa de Parto e avaliar a satisfação das gestantes com o cuidado pré-natal.

\section{MÉTODO}

Estudo descritivo de abordagem quantitativa cujo delineamento se baseou na pesquisa de opinião, que visa conhecer as atitudes, os pontos de vista e preferências de uma dada amostra da população.

O cenário do estudo foi uma Casa de Parto localizada no município do Rio de Janeiro, que é uma unidade pública de saúde. Ela tem uma estrutura física plana semelhante a uma casa, com jardinagem no pátio externo e ambientes coloridos com decoração harmoniosa e suave. Possui consultório de atendimento pré-natal, sala de preparo de medicações e três suítes (com uma ampla cama de casal, berço e toalete individualizado), que contem todo o instrumental recomendado, além dos recursos para cuidado e conforto durante o trabalho de parto (bola, banquinho e banheira, entre outros).

Dispõe de uma sala de cuidados com leito obstétrico do tipo Pré-Parto, Parto e Puerpério (PPP) e berço aquecido para o primeiro atendimento ao neonato; sala de estar 


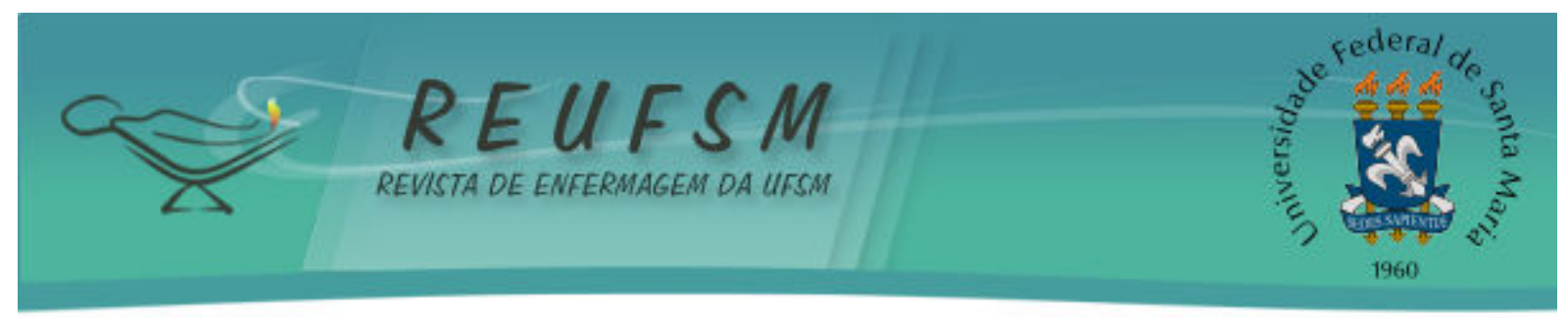

para familiares e gestantes com aparelho de TV; sala para oficinas educativas com aparelhos audiovisuais e uma ambulância para os casos que necessitam de remoção para a maternidade de referência, nas vinte e quatro horas.

Além destas características organizacionais, a coordenação da Casa de Parto é exercida por enfermeiras obstétricas, responsáveis pela administração e condução técnica dos cuidados desenvolvidos. Atuam dezoito enfermeiras obstétricas, oito técnicos de enfermagem, uma nutricionista, uma assistente social e trabalhadores terceirizados para os serviços de cozinha, limpeza, segurança e de ambulância.

As participantes da pesquisa foram gestantes inscritas no atendimento pré-natal da Casa de Parto e que realizaram seis ou mais consultas. Para atender estes critérios, houve consulta prévia da agenda de marcação de consultas e dos registros do prontuário para verificação do número de consultas realizadas. Nesta verificação, não foram considerados os registros dos atendimentos das intercorrências obstétricas e daqueles realizados por outros profissionais no curso do acompanhamento pré-natal, como da assistente social e da nutricionista.

Posteriormente a essa verificação, as gestantes selecionadas foram convidadas para participar do estudo após a consulta pré-natal da enfermeira obstétrica. Aquelas que tiveram indicação de internação para o parto ou de encaminhamento para maternidade referência foram excluídas do estudo.

De acordo com os dados oficiais da SMS/RJ, nos anos de 2007, 2008 e 2009 foram matriculadas 2198 mulheres no acompanhamento pré-natal da Casa de Parto. Destas, 891 gestantes foram referenciadas para outras unidades de saúde, totalizando 1307 grávidas atendidas no pré-natal nestes três anos. Este quantitativo corresponde à média anual de 436 gestantes cadastradas.

Esses dados da população representam o quantitativo global das gestantes matriculadas no pré-natal, que inclui as mulheres que estavam no primeiro, segundo e terceiro trimestres de gravidez. Como não há estratificação pelo trimestre de gestação, era desconhecido o quantitativo de gestantes no terceiro trimestre. Nesta fase da gravidez é esperado que as mulheres tenham realizado maior número de consultas de pré-natal em comparação com aquelas que estão no primeiro e segundo trimestres de gestação. Portanto, optou-se por uma amostragem não probabilística, por conveniência.

Na produção de dados foi utilizado questionário estruturado e constituído de duas partes, ambas com questões objetivas e fechadas. A primeira parte foi composta por questões atinentes à caracterização das gestantes: faixa etária, paridade e número de consultas pré-natal.

A segunda parte do instrumento contemplou as variáveis relativas ao objeto do estudo: os motivos da escolha pelo atendimento na Casa de Parto e satisfação com o cuidado pré-natal (acompanhamento pré-natal; tempo de espera e qualidade do atendimento nas consultas; disponibilidade da enfermeira em responder as dúvidas; atenção e cordialidade das enfermeiras e da equipe de profissionais; acolhimento do companheiro e familiares) e com a ambiência (privacidade durante o atendimento, conforto das instalações, limpeza e organização do ambiente).

$\mathrm{Na}$ investigação dos motivos da escolha pelo serviço de saúde, as participantes do estudo poderiam marcar até duas afirmativas descritas no questionário. As questões que intentavam a mensuração da satisfação foram baseadas na escala de Likert, que utiliza cinco categorias ordinais. Estas categorias recebem valores de cinco a um e correspondem ao maior e ao menor nível de satisfação, respectivamente. Deste modo, as categorias receberam a seguinte valoração: 5- Muito Satisfeita (MS); 4- Satisfeita (S); 3- Neutra (N) ou indiferente (nem satisfeita e nem insatisfeita); 2- Insatisfeita (I) e 1 - Muito Insatisfeita (MI). 


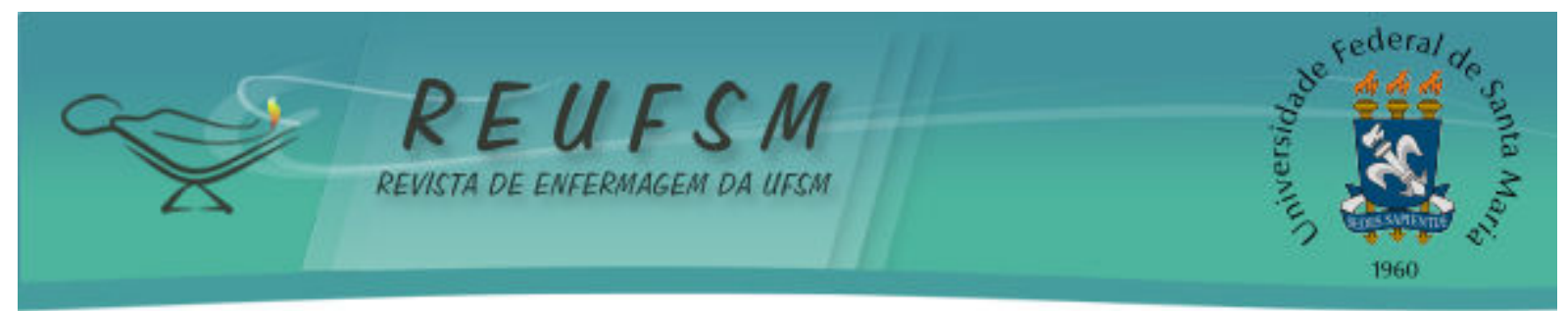

Os dados foram coletados no período de janeiro a março de 2011. Em seguida foram armazenados e tabulados em planilha Microsoft Excel ${ }^{\circledR}$. Inicialmente, receberam o tratamento estatístico descritivo, com suas frequências absolutas e relativas. Nos dados relativos à satisfação foi realizada análise fatorial por meio do cálculo da média ponderada de cada item, o somatório da multiplicação entre a frequência e o valor da resposta na escala de Likert. Em seguida, foi calculada a razão entre esta média e o número total de sujeitos, que resultou no Ranking Médio (RM) das variáveis do objeto em estudo.

A pesquisa obteve parecer favorável do Comitê de Ética em Pesquisa da SMS-RJ, protocolo $n^{\circ} 202 A / 2010$, cujos procedimentos éticos foram observados em todas as suas fases de execução, conforme preconizava a resolução CNS n¹96/96, vigente na época em que o estudo foi realizado.

\section{RESULTADOS E DISCUSSÃO}

Foram $51(100 \%)$ gestantes incluídas no estudo e que responderam ao instrumento de coleta de dados. A amostra estudada representou $11,7 \%$ da média anual de todas as gestantes cadastradas no pré-natal da Casa de Parto.

As gestantes em acompanhamento pré-natal na Casa de Parto eram predominantemente mulheres jovens. Metade $(26 ; 50,1 \%)$ estava na faixa etária de 20 a 24 , seguida do grupo que inclui as adolescentes, com idade variando entre 15 a 19 anos $(11 ; 21,6 \%)$, e daquelas na faixa etária entre 25 a 29 anos $(10 ; 19,6 \%)$. Apenas uma $(1,9 \%)$ gestante tinha a idade acima de 35 anos.

Predominaram as mulheres que realizaram 6 a 7 consultas de pré-natal $(33 ; 64,7 \%)$ e aquelas com 8 a 9 consultas realizadas $(13 ; 25,5 \%)$, bem como aquelas que eram nulíparas $(32 ; 62,8 \%)$. As que tinham dois ou mais partos anteriores representaram $25,5 \%$ (13) do total da amostra investigada.

A proporção de nulíparas que realizava o pré-natal na Casa de Parto foi maior que aquela encontrada entre as parturientes assistidas em dois CPN brasileiros, $45,4 \%$ e $46,3 \% .{ }^{12-13}$ Quanto à predominância de gestantes que realizaram 6 a 7 consultas de prénatal, esta é uma tendência da assistência pré-natal no município do Rio de Janeiro. Neste município, a maioria das gestantes realizam o quantitativo de consultas pré-natais preconizado pelo Ministério da Saúde, pelo menos seis consultas. ${ }^{14} \mathrm{Na}$ avaliação dos dados da saúde no Brasil, a proporção de nascimentos cujas mães realizaram sete ou mais consulta passou de 46\%, em 2000, para 61,1\%, em 2010. Apenas os nascimentos ocorridos nas regiões norte e nordeste apresentaram percentuais menores que a taxa brasileira para este parâmetro em $2010,37 \%$ e $45,5 \%$, respectivamente. ${ }^{5}$

O número de consultas realizadas tem relação com a satisfação com a assistência pré-natal e o desfecho perinatal. Revisão sistemática evidenciou que há diminuição na satisfação das mulheres quando o modelo de atenção pré-natal adota menor número de consultas para gestações de baixo risco obstétrico, menos de cinco consultas nos países cuja população tem menor renda. Nestes países, o modelo de atenção com consultas reduzidas foi associado com o aumento da mortalidade perinatal. ${ }^{15}$

As gestantes obtiveram informações acerca da Casa de Parto por meio de conhecidos e amigos (27; 52,9\%) e familiares (22; 43,1\%), cuja indicação decorreu da própria vivência ou da experiência de pessoas do convívio social com o atendimento nesta instituição. Os principais motivos da escolha da Casa de Parto para o acompanhamento pré-natal foram: possuir ambiente acolhedor $(20 ; 39,2 \%)$; permitir a presença do acompanhante no parto $(12 ; 23,5 \%)$; incentivar o parto normal $(11 ; 21,6 \%)$; atender de forma humanizada $(10 ; 19,6 \%)$; possibilitar a escolha pelo tipo de parto normal $(8 ; 13,7 \%)$ e 


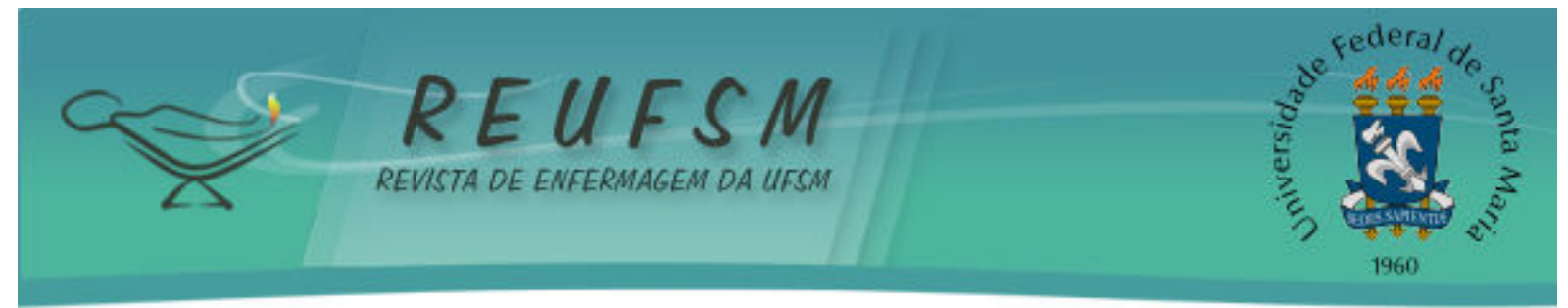

garantir a internação no momento do parto $(7 ; 13,7 \%)$. Estes dados podem ser visualizados na Tabela 1.

Tabela 1 - Distribuição das gestantes $(n=51)$ segundo os motivos da escolha pelo local do acompanhamento pré-natal. Casa de Parto, Rio de Janeiro-RJ. 2011

\begin{tabular}{lcc}
\hline Motivos & $\mathbf{n}$ & $\%$ \\
\hline Possuir ambiente acolhedor & 20 & 39,2 \\
Permitir a presença do acompanhante no parto & 12 & 23,5 \\
Incentivar o parto normal & 11 & 21,6 \\
Atender de forma humanizada & 10 & 19,6 \\
Possibilitar a escolha do tipo de parto normal & 8 & 15,7 \\
Garantir a internação no momento do parto & 7 & 13,7 \\
Contar com atendimento profissional qualificado & 6 & 11,8 \\
Dispor de ambulância nas 24 h & 4 & 7,8 \\
Estar mais próxima da residência & 3 & 5,9 \\
Ser atendida com hora marcada & 2 & 3,9 \\
\hline
\end{tabular}

$\left(^{*}\right)$ Parto nas posições vertical, lateral, de cócoras, sentada no banquinho de parto, semi-sentada na banheira ou na cama da suíte de parto.

A indicação do serviço para outras pessoas pode ser um parâmetro complementar na avaliação da qualidade da assistência do serviço de saúde. Pesquisa avaliativa realizada em sete municípios brasileiros identificou que há fatores subjetivos relacionados à escolha do local de realização do acompanhamento pré-natal, como a percepção da qualidade do serviço prestado e a necessidade de segurança no momento do parto. Dentre os fatores relacionados com esta escolha, há a experiência relatada por familiar ou conhecido que foi atendido previamente no local e a possibilidade de ser assegurada a referência para o parto. ${ }^{16}$

As recomendações fornecidas por profissionais que atuam no pré-natal e por parentes que tiveram experiências positivas foram consideradas importantes na opção das mulheres pelo atendimento em CPN no momento da parturição. ${ }^{17}$ Portanto, houve convergência dos resultados encontrados para este parâmetro no presente estudo. Este achado sugere que a percepção positiva da clientela acerca da assistência prestada tem influência na opção das gestantes pela Casa de Parto como cenário do cuidado do pré-natal e do parto.

Em quase a totalidade das variáveis avaliadas houve maior frequência na resposta do escore 5, muito satisfeita, da escala de Likert. Portanto, o Ranking Médio foi elevado, maior que 4, no nível de satisfação referente aos seguintes dados: acompanhamento pré-natal em geral $(R M=4,65)$; qualidade do atendimento das consultas $(R M=4,53)$; disponibilidade da enfermeira em responder as dúvidas $(\mathrm{R} M=4,51)$; atenção e cordialidade das enfermeiras $(\mathrm{RM}=4,82)$; atenção e cordialidade da equipe de profissionais $(\mathrm{RM}=4,69)$; acolhimento do companheiro e familiares $(R M=4,65)$; privacidade durante 0 atendimento $(R M=4,53)$; conforto das instalações $(R M=4,65)$ e limpeza e organização do ambiente $(R M=4,51)$.

Na variável "tempo de espera para as consultas" houve maior proporção (28; $54,9 \%)$ no escore 4 (satisfeita) e a única variável em que houve frequência nas respostas "insatisfeita" e "muito insatisfeita", que resultou no RM de 3,63, conforme descrito na Tabela 2. 


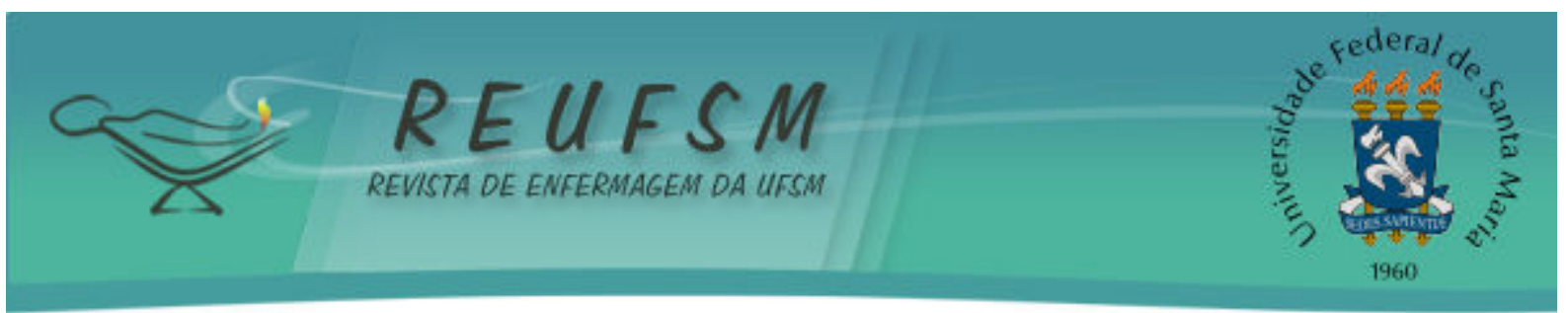

Tabela 2 - Avaliação da satisfação das gestantes com o cuidado pré-natal. Casa de Parto, Rio de Janeiro-RJ. 2011 ( $\mathrm{n}=51)$.

\begin{tabular}{|c|c|c|c|c|c|c|c|}
\hline Variáveis & $\begin{array}{c}M S^{(*)} \\
(5) \\
N \quad(\%) \\
\end{array}$ & $\begin{array}{c}S \\
(4) \\
N(\%) \\
\end{array}$ & $\begin{array}{c}N \\
(3) \\
N(\%) \\
\end{array}$ & $\begin{array}{c}\text { I } \\
(2) \\
N(\%) \\
\end{array}$ & $\begin{array}{c}\text { MI } \\
(1) \\
N(\%) \\
\end{array}$ & $\begin{array}{c}\text { Não } \\
\text { respondeu } \\
\mathrm{N}(\%) \\
\end{array}$ & $\begin{array}{c}\text { Ranking } \\
\text { Médio } \\
\text { (RM) }\end{array}$ \\
\hline $\begin{array}{l}\text { Acompanhamento pré-natal } \\
\text { em geral }\end{array}$ & $33(64,7)$ & $18(35,3)$ & & & & & 4,65 \\
\hline $\begin{array}{l}\text { Tempo de espera para as } \\
\text { consultas }\end{array}$ & $9(17,7)$ & $28(54,9)$ & $4(7,8)$ & $6(11,8)$ & $4(7,8)$ & & 3,63 \\
\hline $\begin{array}{l}\text { Qualidade do atendimento } \\
\text { nas consultas }\end{array}$ & $27(52,9)$ & $24(47,1)$ & & & & & 4,53 \\
\hline $\begin{array}{l}\text { Disponibilidade da } \\
\text { enfermeira em responder as } \\
\text { dúvidas }\end{array}$ & $30(58,8)$ & $20(39,2)$ & & & & $1(2,0)$ & 4,51 \\
\hline $\begin{array}{l}\text { Atenção e cordialidade das } \\
\text { enfermeiras }\end{array}$ & $42(82,4)$ & $9(17,6)$ & & & & & 4,82 \\
\hline $\begin{array}{l}\text { Atenção e cordialidade da } \\
\text { equipe de profissionais }\end{array}$ & $39(76,5)$ & $11(21,5)$ & & & & $1(2,0)$ & 4,69 \\
\hline $\begin{array}{l}\text { Acolhimento do companheiro } \\
\text { e familiares }\end{array}$ & $33(64,7)$ & $18(35,3)$ & & & & & 4,65 \\
\hline $\begin{array}{lll}\text { Privacidade } & \text { durante } & \text { o } \\
\text { atendimento } & & \end{array}$ & $27(52,9)$ & $24(47,1)$ & & & & & 4,53 \\
\hline Conforto das instalações & $33(64,7)$ & $18(35,3)$ & & & & & 4,65 \\
\hline $\begin{array}{l}\text { Limpeza e organização do } \\
\text { ambiente }\end{array}$ & $30(58,8)$ & $20(39,2)$ & & & & $1(2,0)$ & 4,51 \\
\hline
\end{tabular}

$\left(^{*}\right)$ Legenda: $M S=$ Muito Satisfeita; S= Satisfeita; N= Neutra; I= Insatisfeita e MI= Muito Insatisfeita.

Em relação aos resultados de estudo sobre o atendimento em CPN, a satisfação da clientela também foi relacionada com o ambiente agradável da instituição em comparação com o hospital; disponibilidade de contar com o acompanhante; presença contínua dos profissionais no ambiente de cuidado; respeito à liberdade de escolha; fornecimento de respostas aos questionamentos; existência de apoio emocional; respeito à fisiologia do parto, entre outros. ${ }^{17}$

Apesar dessas características institucionais exercerem influência na opção da mulher pelo atendimento nos CPN e Casa de Parto, o presente estudo encontrou pequeno quantitativo (4\%) de gestantes referenciadas pelas unidades da rede básica para 0 acompanhamento pré-natal na Casa de Parto. As causas deste referenciamento reduzido necessitam ser esclarecidas. As próprias características da instituição podem estar envolvidas nesta questão. Há segmentos sociais e profissionais que questionam a conformação institucional da Casa de Parto devido à ausência de médicos e à presunção de insegurança assistencial quando comparada ao ambiente hospitalar. Estes questionamentos têm mobilizado as entidades sociais em defesa da humanização do parto e do direito de escolha mulher por locais diferenciados do hospital. ${ }^{18}$

A cultura assistencial hegemônica no campo obstétrico brasileiro caracteriza-se pela superestimação dos benefícios da tecnologia e na subestimação ou na negação dos desconfortos e efeitos adversos das intervenções. Apesar deste contexto predominante, as mulheres usuárias da Casa de Parto manifestaram alta satisfação pelo cuidado pré-natal.

A atenção pré-natal deve ser qualificada e humanizada e promover o acolhimento e a escuta da mulher e de seu acompanhante. Tal perspectiva pressupõe o 


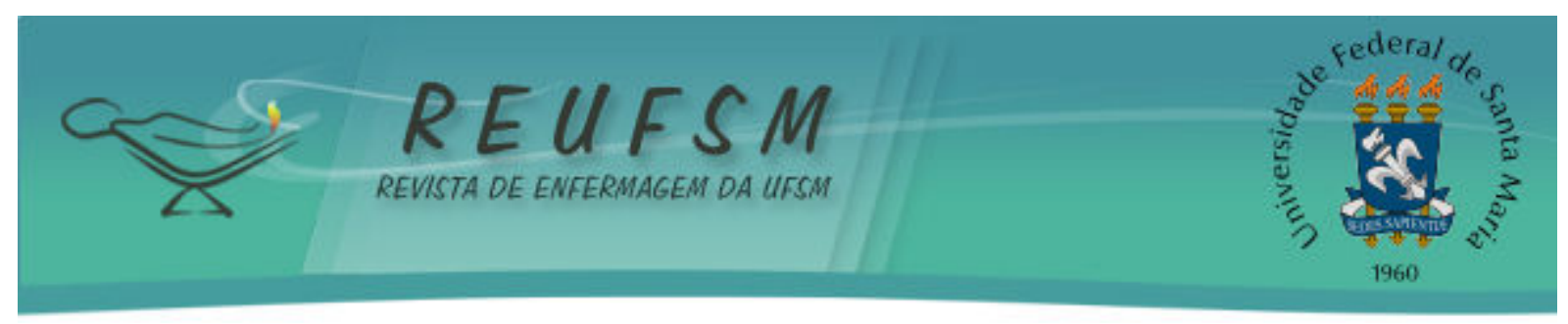

estabelecimento da relação de cuidado, que implica em resgatar a subjetividade, assegurar direitos inalienáveis e construir relações humanas democráticas, superando as assimetrias de poder que ainda permeiam a sociedade, em particular na assistência à saúde da mulher.

Estudos sobre a satisfação com o acompanhamento pré-natal, tanto em unidade da rede básica quanto em Casa de Parto, evidenciaram que o cuidado de enfermagem é baseado na escuta, diálogo, atenção, respeito e confiança. ${ }^{7,9-11}$

Os achados dessas investigações têm similaridade com os indicadores de satisfação encontrados na presente pesquisa, que também estiveram relacionados com as características do cuidado. O cuidado é determinante nas configurações das práticas assistenciais e da relação profissional-cliente, que exercem influência no nível de satisfação das gestantes investigadas na presente pesquisa.

Esta convergência de achados pertinentes às características relatadas pelas mulheres acerca da assistência de enfermagem, tanto em unidades da rede básica quanto em Casa de Parto, pode representar a existência de elementos comuns e distintivos de um paradigma profissional subjacente.

Pesquisa fenomenológica desvelou que o cuidado das enfermeiras obstétricas à mulher na Casa de Parto é singular. 0 cuidado não faz pela mulher, mas potencializa sua capacidade de reconhecer-se e cuidar de si. É um cuidado que se revela desde o pré-natal e continua no pós-parto, sendo uma construção fundada no compartilhar, que envolve a enfermeira e a mulher, e também na reciprocidade, solidariedade e liberdade. É um cuidado autêntico com as coisas do cotidiano e, neste encontro, a enfermeira-é-com-amulher e cria um espaço de abertura para o ser-no-mundo da mulher. Portanto, é um cuidado que tem uma identidade: é da enfermeira para a mulher. ${ }^{19}$

Ao se analisar o exercício da autonomia da parturiente durante o parto normal assistido pela enfermeira obstétrica em Casa de Parto, revela-se a importância do cuidado com competência técnica, humana e ética, no respeito às necessidades, desejos, expectativas e escolhas para a satisfação das mulheres no processo da gestação e parto. ${ }^{20}$ Portanto, o cuidado de enfermagem obstétrica neste cenário assistencial pode efetivar vivências positivas e prazerosas da gestação, parto e nascimento, o que contribui para a reversão do imaginário sociocultural de boa parte das mulheres-grávidas e de seus familiares, que relaciona estes eventos vitais ao medo, à dor e ao sofrimento. ${ }^{10}$

Apesar das mulheres manifestarem alto nível de satisfação com o cuidado prénatal na Casa de Parto, o menor escore de satisfação foi atinente ao tempo de espera para as consultas. Embora não esteja situado na faixa de insatisfação, este resultado aponta para a necessidade de melhoria neste aspecto.

A Casa de Parto tem como proposta assistencial realizar consultas individualizadas, que buscam atender as necessidades de cuidado e promover a participação ativa da mulher. Portanto, as consultas de pré-natal são planejadas para que tenham uma maior duração, cerca de cinquenta minutos a consulta de primeira vez e trinta minutos as consultas subsequentes.

Além dessa diferenciação organizacional, a equipe diária de enfermeiras obstétricas divide-se entre os cuidados ao pré-natal, parto, pós-parto e prontoatendimento, assim como na função de preceptoria das residentes de enfermagem obstétrica. Portanto, os resultados encontrados indicam ser necessário o ajuste do agendamento diário de consultas frente a esta dinâmica assistencial e educacional para melhoria desse indicador da qualidade assistencial. 


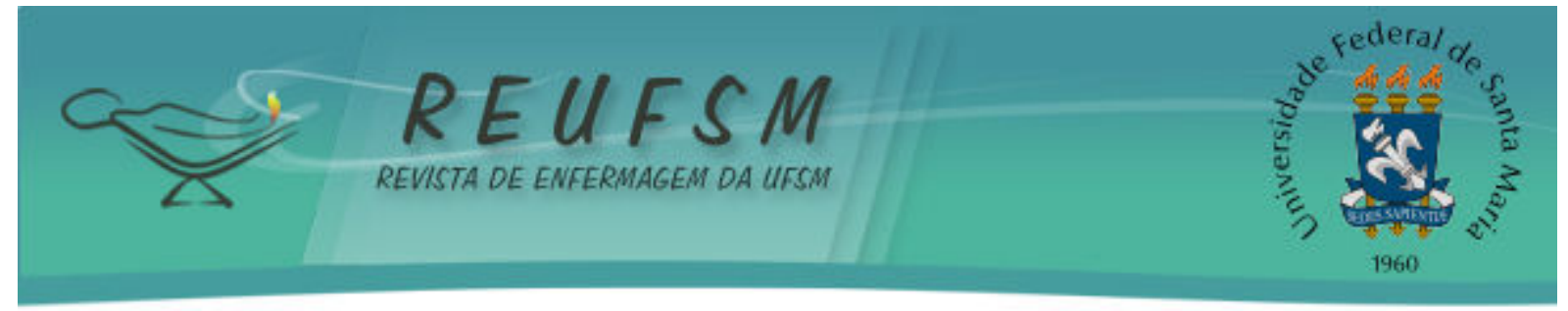

CONCLUSÃO

As gestantes participantes do estudo eram mulheres jovens, predominantemente nulíparas, e que realizaram mais de seis consultas de pré-natal. Elas optaram pela assistência na Casa de Parto devido ao seu ambiente acolhedor, à garantia da presença do acompanhante e da internação no momento do parto, ao incentivo ao parto normal e à humanização da assistência.

As mulheres apresentaram alto nível de satisfação com o cuidado pré-natal na Casa de Parto, sobretudo com a qualidade da atenção e cordialidade profissional; acolhimento do companheiro e familiares; privacidade e conforto do ambiente de cuidado.

A satisfação com o tempo de espera para as consultas foi menor em comparação com os demais indicadores, o que denota a importância da revisão periódica dos fluxos do atendimento a fim de garantir a manutenção da qualidade da assistência oferecida.

Apesar dos resultados da pesquisa não serem generalizáveis, em virtude do tamanho da amostra e de serem restritos ao grupo estudado e ao local onde a mesma foi realizada, eles trouxeram elucidações preliminares acerca da qualidade do cuidado de enfermagem obstétrica em Casa de Parto, que têm convergência com os achados descritos em estudos internacionais acerca deste cenário assistencial.

O tema de investigação ainda requer avanços, necessários para construir um arcabouço científico sólido acerca da assistência de enfermagem obstétrica em Casa de Parto e suas possibilidades de contribuir para a superação dos desafios atinentes à incorporação do modelo de cuidado humano, sensível e ético nas unidades de atenção obstétrica do país.

\section{REFERÊNCIAS}

1. Pereira ALF, Moura MAV. Hegemonia e contra-hegemonia no processo de implantação da Casa de Parto no Rio de Janeiro. Rev Esc Enferm USP. 2009 dez;43(4):872-9.

2. Hodnett ED, Downe S, Walsh D, Weston J. Alternative versus conventional institutional settings for birth. Cochrane Database Syst Rev. 2010 Sep 8;(9):CD000012.

3. Bergström M. Continuous support in labor has beneficial effects for mother and baby. Evid Based Med. 2011 Dec;16(6):182-3.

4. Brasil. Ministério da Saúde. PNASS - Programa Nacional de Avaliação de Serviços de Saúde. Brasília: Ministério da Saúde; 2007.

5. Brasil. Ministério da Saúde. Saúde Brasil 2011: uma análise da situação de saúde e a vigilância da saúde da mulher. Brasília: Ministério da Saúde; 2012.

6. Costa GD, Cotta RMM, Reis JR, Batista RS, Gomes AP, Franceschini SCC. Avaliação o cuidado à saúde da gestante no contexto do Programa Saúde da Família. Ciênc Saúde Coletiva. 2009 set/out;14 Supl 1:1347-57.

7. Barbosa TLA, Gomes LMX, Dias OV. O pré-natal realizado pelo enfermeiro: a satisfação das gestantes. Cogitare Enferm. 2011 jan/mar;16(1):29-35.

8. Guerreiro EM, Rodrigues DP, Silveira MAM, Lucena NBF. Cuidado pré-natal na atenção básica de saúde sob o olhar de gestantes e enfermeiros. REME Rev Min Enf. 2012 jul/set;16(3):315-23.

9. Santos AL, Radovanovic CAT, Marcon SS. Assistência pré-natal: satisfação e expectativas. Rev RENE. 2010 dez;11(N Esp):61-71. 
10. Velasque EAG, Pradebon VM, Cabral FB. O enfermeiro no processo parir/nascer: estratégia de cuidado e humanização do parto. Rev Enferm UFSM [Internet]. 2011 [acesso em 2014 mar 10];1(1):807. Disponível em: http://cascavel.ufsm.br/revistas/ojs-2.2.2/index.php/reufsm/article/view/1991/1514.

11. Seibert SL, Gomes ML, Vargens OMC. Assistência pré-natal da Casa de Parto do Rio de Janeiro. Esc Anna Nery Rev Enferm. 2008 dez;12(4):758-64.

12. Schneck CA, Riesco MLG, Bonadio IC, Diniz CSG, Oliveira SMJV. Resultados maternos e neonatais em centro de parto normal peri-hospitalar e hospital. Rev Saúde Pública. 2012 fev;46(1):77-86.

13. Lobo SF, Oliveira SMJV, Schneck A, Silva FMB, Bonadio IC, Riesco MLG. Resultados maternos e neonatais em Centro de Parto Normal peri-hospitalar na cidade de São Paulo, Brasil. Rev Esc Enferm USP. 2010;44(3):812-8.

14. Lopes FO, Oliveira MIC, Brito AS, Fonseca VM. Fatores associados ao uso de suplementos em recém-natos em alojamento conjunto no município do Rio de Janeiro, 2009. Ciênc Saúde Coletiva. 2013 fev;18(2):431-9.

15. Dowswell T, Carroli G, Duley L, Gates S, Gülmezoglu AM, Khan-Neelofur D, et al. Alternative versus standard packages of antenatal care for low-risk pregnancy. Cochrane Database of Syst Rev. 2010;(10):CD000934.

16. Almeida CAL, Tanaka OY. Perspectiva das mulheres na avaliação do Programa de Humanização do Pré-Natal e Nascimento. Rev Saúde Pública. 2009 fev;43(1):98-104.

17. Jamas MT, Hoga LA, Tanaka AC. Mothers' birth care experiences in a Brazilian birth centre. Midwifery. 2011 Oct;27(5):693-9.

18. Diniz SG. Women's movement defends birth centres in Brazil. Reprod Health Matters. 2009 nov;17(34):188-9.

19. Zveiter M. O cuidado de enfermeiras obstétricas com-a-mulher-que-dá-à-luz-na-Casade-Parto: uma hermenêutica em Heidegger [tese]. Rio de Janeiro: Universidade Federal do Rio de Janeiro, Escola de Enfermagem Anna Nery; 2011. 112 p.

20. Pereira ALF, Bento AD. Autonomia no parto normal na perspectiva das mulheres atendidas na casa de parto. Rev RENE. 2011 jul/set;12(3):471-7.

Data de recebimento: 23/03/2014

Data de aceite: 26/09/2014

Contato com autor responsável: Adriana Lenho de Figueiredo Pereira

Endereço postal: Boulevard 28 de Setembro, 157, sala 824. Vila Isabel. Rio de Janeiro - RJ. CEP. 20551-030

E-mail: adrianalenho.uerj@gmail.com 